\title{
Erythropoese
}

\section{Ex vivo-Expansion von roten Blutzellen aus humanen Stammzellen}

ISABEL DORN, CLAUDIA BERNECKER

UNIVERSITÄTSKLINIK FÜR BLUTGRUPPENSEROLOGIE UND TRANSFUSIONSMEDIZIN, MEDIZINISCHE UNIVERSITÄT GRAZ, ÖSTERREICH

\section{The growing demand of blood products in modern medicine causes} chronic limitations in blood supply. Current research focuses on the ex vivo production of red blood cells (RBCs) from different sources including induced pluripotent stem cells (iPSCs). RBC generation from iPSCs is limited by low efficiency so far. Our group established a protocol that enables for higher cellular output and improved enucleation based on closer mimicking of the physiological microenvironment in which erythropoiesis occurs.

DOI: $10.1007 / \mathrm{s} 12268-020-1336-\mathrm{Z}$

(C) Die Autorinnen 2020

Rote Blutkörperchen (Erythrocyten) sind die häufigsten Zellen des peripheren Blutes und transportieren mithilfe des roten Blutfarbstoffes - Hämoglobin - Sauerstoff von der Lunge in alle Gewebe. Ihre Überlebenszeit beträgt ca. 120 Tage. Therapeutisch werden Erythrocyten in Form von Erythrocytenkonzentraten (EK) bei einem Mangel an Sauerstoffträgern eingesetzt. Ein EK enthält ca. $2 \times 10^{12}$ Erythrocyten. Im Rahmen der modernen Hochleistungsmedizin und der zunehmenden Alterung der Menschen nimmt der Bedarf an Transfusionen stetig zu. Dieser wird überwiegend durch freiwillige Blutspenden gedeckt. Bereits heute bestehen chronische Engpässe, insbesondere bei Patienten mit seltenen Blutgruppen oder nach Immunisierung gegen mehrere Erythrocyten-Antigene.

Ex vivo-Kultivierung von Erythrocyten aus humanen hämatopoetischen Stammzellen

Zur Vermeidung solcher Engpässe besteht ein hohes Interesse, Erythrocyten ex vivo im

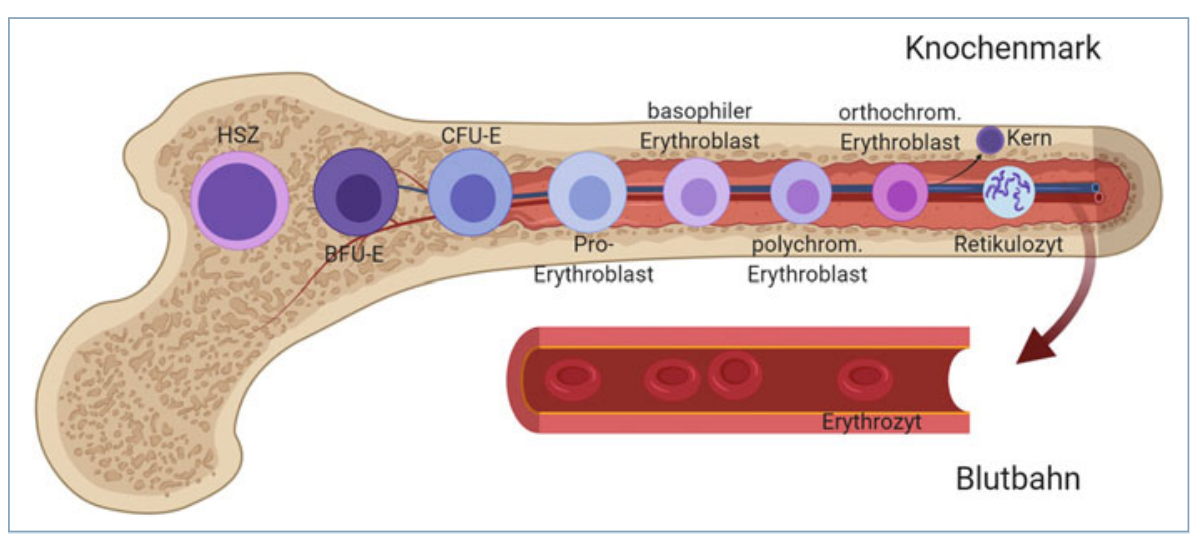

$\Delta$ Abb. 1: Entwicklung von Erythrocyten aus hämatopoetischen Stammzellen (HSZ) im humanen Knochenmark. Im Stadium des orthochromatischen Erythroblasten erfolgt die Ausstoßung des Zellkerns. Der verbleibende Retikulocyt wandert aus dem Knochenmark in das periphere Blut, wo er durch den Abbau restlicher Zellorganellen und Umbau der Zellmembran zum bikonkaven Erythrocyten ausreift. BFU-E: Burst forming unit erythroid, früheste erythropoetische Vorläuferzelle; CFU-E: Colony forming unit erythroid, frühe erythropoetische Vorläuferzelle.
Labor zu generieren. Beim Menschen erfolgt der lebenslange Nachschub an Erythrocyten ausgehend von hämatopoetischen Stammzellen (HSZ) im Knochenmark (Abb. 1). HSZ können in geringem Umfang auch aus dem peripheren Blut isoliert werden. In den letzten Jahren wurden Protokolle etabliert, die es ermöglichen, HSZ bis zum kernlosen Retikulocyten zu differenzieren und dabei im großen Maßstab zu vermehren (bis zu 106-fach). Die Funktionalität von solchen im Labor generierten Erythrocyten konnte in zahlreichen Untersuchungen belegt werden. In der Maus und in einer ersten Proof of PrincipleTransfusion am Menschen weisen kultivierte Erythrocyten eine normale Überlebenszeit auf $[1,2]$. Die vorherrschende Limitation ist jedoch, dass HSZ ex vivo nicht in ihrem undifferenzierten Zustand vermehrt werden können und somit eine limitierte Quelle darstellen.

Ex vivo-Kultivierung von Erythrocyten aus induzierten pluripotenten Stammzellen

Eine mögliche Alternative bietet die Verwendung pluripotenter Stammzellen (PSZ). Da diese prinzipiell unbegrenzt vermehrungsfähig sind, erhofft man sich neben einer noch höheren Expansion den Aufbau von stets verfügbaren Stammzellbanken. Von besonderem Interesse sind induzierte pluripotente Stammzellen (iPS-Zellen), welche durch den Vorgang der Reprogrammierung aus somatischen Zellen des Körpers generiert werden können [3]. Sie ermöglichen zudem die Herstellung Patienten-eigener (autologer) und somit immunologisch unbedenklicher Erythrocyten zum Zwecke der Transfusion. Allerdings ist die Differenzierung von Erythrocyten aus iPS-Zellen sehr komplex, da im Zellkulturmodell zunächst alle Reifungsschritte der Embryonalentwicklung nachvollzogen werden müssen.

Blutbildende Zellen entwickeln sich aus dem mittleren Keimblatt, dem Mesoderm. Während der Embryonal- und Fetalzeit findet die Hämatopoese in örtlich und zeitlich voneinander getrennten Wellen statt. Ausge- 
hend von Zellen im Dottersack entsteht die primitive Hämatopoese, welche der vorrübergehenden Blutversorgung des frühen Embryos dient. Im weiteren Verlauf entstehen in speziellen Bereichen der sich entwickelnden Aorta die ersten HSZ. Diese bilden die Grundlage für die lebenslange definitive Hämatopoese. Die weitere Entwicklung dieser HSZ erfolgt in spezialisierten Gewebsnischen anderer Organe und hängt essenziell von den dort vorherrschenden Umgebungsfaktoren ab. Die „naiven“ HSZ besiedeln zunächst Nischen der Leber und bis zur Geburt das Knochenmark [4].

In den letzten Jahren wurden Protokolle etabliert, die es ermöglichen, Erythrocyten aus iPS-Zellen zu generieren [5, 6]. Allerdings ergeben sich im Vergleich zu adulten HSZ deutliche Limitationen. So konnten bislang nur vergleichsweise geringe Expansionsraten erreicht werden, was möglicherweise an der Umgehung der hämatopoetischen Vorläuferstadien mit hohem Proliferationspotenzial liegt. Während der erythrocytären Differenzierung versagen die Zellen zudem in der Ausstoßung des Zellkerns (die Enukleation liegt unter 20 Prozent) und im Wechsel von fetalem zu dem nach der Geburt vorherrschenden adulten Hämoglobin. Eine mögliche Ursache für diese Limitationen ist das Fehlen der physiologischen Nische, in welcher die Entwicklung von Erythrocyten im Körper stattfindet.

Der Einfluss solcher Gewebsnischen wurde bislang kaum berücksichtigt, insbesondere da die Umgebungs- und Regulationsmechanismen während der Embryonal- und Fetalzeit wenig bekannt sind. Die Induktion der Hämatopoese und Erythropoese erfolgt stattdessen durch eine Vielzahl an Wachstumsfaktoren (bis zu über 15), die während unterschiedlicher Phasen (bis zu neun) eingesetzt werden. Zusätzliche Verdau- und Aufreinigungsschritte zerstören zudem Gewebszusammenhänge und möglicherweise wichtige Zellinteraktionen. Aufgrund der langen Kulturdauer, des umfangreichen Cytokineinsatzes und der häufigen Medienwechsel entstehen zudem sehr hohe Kosten.
Etablierung eines vereinfachten Zellkulturmodells unter Berücksichtigung der physiologischen Nische

Um diese negativen Einflüsse zu reduzieren, hat unsere Arbeitsgruppe ein vereinfachtes Zellkulturmodell zur Generierung von Erythrocyten aus iPS-Zellen etabliert (Abb. 2, [7]). In diesem Modell werden die iPS-Zellen zunächst zur Bildung von embryoid bodies (EBs) angeregt. Um die Induktion des Mesoderms und der Hämatopoese zu fördern, erfolgt eine Stimulation mit nur drei Cytokinen (Stammzellfaktor [SCF], Erythropoietin und Interleukin-3 [IL-3]), von denen bekannt ist, dass sie die Hämatopoese und Erythropoese positiv beeinflussen [8, 9]. Der Mediumwechsel erfolgt nur einmal pro Woche, sodass parakrine Faktoren erhalten bleiben und gleichzeitig der Mediumverbrauch und somit die Kosten deutlich reduziert werden. Im Folgenden adhärieren die EBs an der Oberfläche der Kulturschale und bilden dreidimensionale Sphäroide aus, die von einem Netzwerk aus Stromazellen umgeben sind. Von diesen zellulären Komplexen - hemato- 


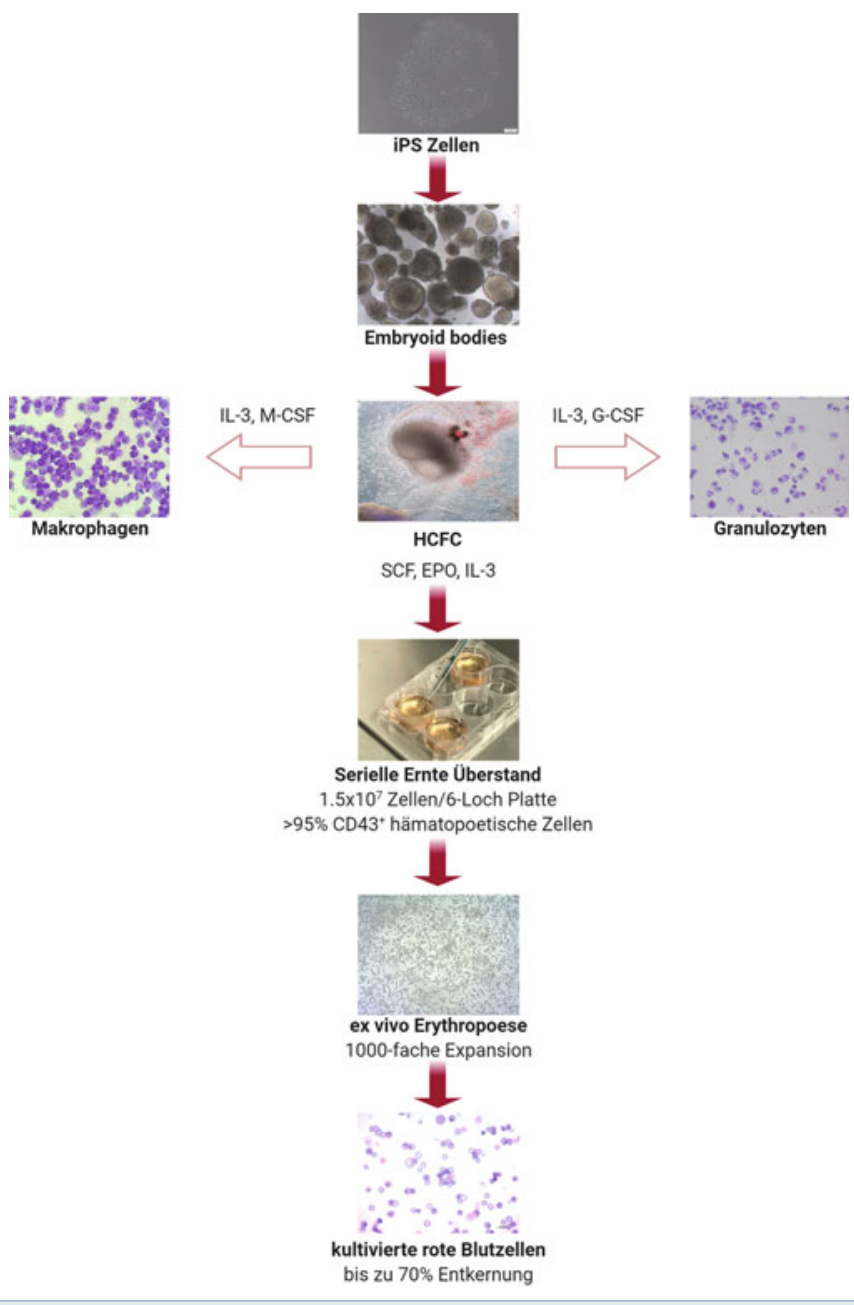

A Abb. 2: Schematische Darstellung des neu etablierten Modells zur ex vivo-Generierung von Erythrocyten aus humanen induzierten pluripotenten Stammzellen (iPS-Zellen). Embryoid bodies adhärieren unter Stimulation mit Stammzellfaktor (SCF), Erythropoetin (EPO) und Interleukin-3 (IL-3) an die Kulturschale und entwickeln komplexe Sphäroide, umgeben von Stromazellen (HCFC, hematopoietic cell forming complex). Vom HCFC werden im weiteren Verlauf kontinuierlich $\mathrm{CD}_{4} 3^{+}$ hämatopoetische Zellen in den Überstand sezerniert und können wiederholt für die weitere erythrocytäre Differenzierung geerntet werden. Durch Stimulation des HCFC mit G-CSF/IL-3 oder M-CSF/IL-3 lassen sich alternativ Granulocyten bzw. Makrophagen erzeugen.

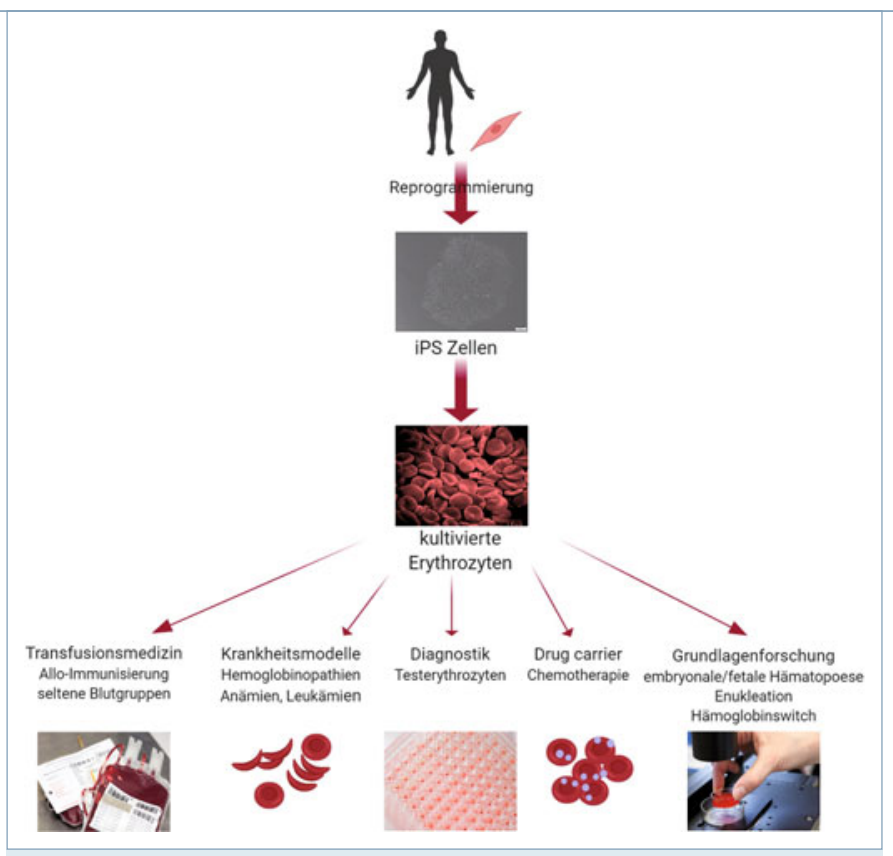

A Abb. 3: Schematische Darstellung zukünftiger Einsatzmöglichkeiten von aus induzierten pluripotenten Stammzellen (iPS-Zellen) generierten Erythrocyten. In der Transfusionsmedizin können ex vivo kultivierte Erythrocyten zur Herstellung von (autologen) Erythrocytenkonzentraten und Testerythrocyten verwendet werden. Darüber hinaus erlauben iPS-Zellen neben der Grundlagenforschung, z. B. zur primitiven und fetalen Erythropoese, die Abbildung erythrocytärer Erkrankungen, wie z. B. der Sichelzellenanämie, in der Kulturschale. Ein weiteres innovatives Gebiet ist die Verwendung von Erythrocyten als drug carrier.

werden (bis zu 70 Prozent). Insgesamt können über 34.000 erythrocytäre Zellen aus einer iPS-Zelle generiert werden. Unsere Studien geben somit Hinweise darauf, dass zelluläre Interaktionen, die bereits in frühen Entwick-

poietic cell forming complex (HCFC) genannt - werden im weiteren Verlauf hämatopoetische Zellen in einer Reinheit von über 95 Prozent kontinuierlich in den Überstand ausgestoßen, welche über einen Zeitraum von ca. sechs Wochen regelmäßig für die weitere erythrocytäre Differenzierung abgeschöpft werden können.

Die initial in den Überstand sezernierten Zellen zeigen zu 70 Prozent phänotypische Charakteristika von HSZ (Expression von CD43, CD34 und CD45) und sind in der Lage, in geeigneten Medien hämatopoetische Kolonien zu bilden. Somit durchlaufen sie das hochproliferative Vorläuferstadium, welches in bisherigen Modellen kaum induziert werden konnte. Während der weiteren Differenzierung zu roten Blutzellen können zudem deutlich höhere Entkernungsraten erzielt lungsstadien stattfinden, wichtig sind für die weitere hämatopoetische und erythrocytäre Differenzierung einschließlich der Enukleation. Die Identifizierung beteiligter Faktoren und zellulärer Interaktionen innerhalb des HCFC sind Gegenstand aktueller Untersuchungen. Darüber hinaus kann das Zellkultursystem durch Änderung des Cytokincocktails (IL-3, G-SCF/M-CSF) auch zur kontinuierlichen Generierung von Monocyten und Makrophagen verwendet werden [10].

\section{Zukünftige Einsatzmöglichkeiten ex vivo kultivierter Erythrocyten}

Ex vivo generierte Erythrocyten können in Zukunft nicht nur im Rahmen der Transfusion und der Grundlagenforschung wertvoll genutzt werden, sondern auch für andere innovative Ansätze (Abb. 3). Hierzu zählt die
Herstellung von Testerythrocyten, die im Labor zur Bestimmung von Blutgruppen genutzt und bislang von ausgesuchten Spendern mühsam zusammengestellt werden. Zunehmend in den Fokus der Wissenschaft gerät die Verwendung von Erythrocyten als drug carrier, um beispielsweise toxische Einflüsse zu reduzieren oder Medikamente kontinuierlich, gesteuert über die Halbwertszeit von Erythrocyten, abzugeben. iPS-Zellen als Ausgangsmaterial bieten zudem die Möglichkeit der Expansion krankheitsspezifischer Zellen im Rahmen von Krankheitsmodellen (z. B. Anämien oder Leukämien). Hierfür können iPS-Zellen der Patienten generiert und als Stammzellbank angelegt werden.

\section{Literatur}

[1] Giarratana MC, Rouard H, Dumont A et al. (2011) Proof of principle for transfusion of in vitro-generated red blood cells. Blood 118:5071-5079

[2] Zeuner A, Martelli F, Vaglio S et al. (2012) Concise review: stem cell-derived erythrocytes as upcoming players in blood transfusion. Stem Cells 30:1587-1596

[3] Takahashi K, Tanabe K, Ohnuki M et al. (2007) Induction of pluripotent stem cells from adult human fibroblasts by defined factors. Cell 131:861-872

[4] Palis J (2014) Primitive and definitive erythropoiesis in mammals. Front Physiol 5:3

[5] Ackermann M, Liebhaber S, Klusmann JH et al. (2015) Lost in translation: pluripotent stem cell-derived hematopoiesis. EMBO Mol Med 7:1388-1402

[6] Focosi D, Amabile G (2017) Induced pluripotent stem cell-derived red blood cells and platelet concentrates: from bench to bedside. Cells 7, doi: 10.3390/cells7010002

[7] Bernecker C, Ackermann M, Lachmann N et al. (2019) Enhanced ex vivo generation of erythroid cells from human 
induced pluripotent stem cells in a simplified cell culture system with low cytokine support. Stem Cells Dev 28:1540-1551 [8] Douay L, Giarratana MC, Drouet X et al. (1991) The role of recombinant haematopoietic growth factors in human longterm bone marrow culture in serum-free medium. $\mathrm{Br} \mathrm{J}$ Haematol 79:27-32

[9] Wu H, Klingmüller U, Besmer P et al. (1995) Interaction of the erythropoietin and stem-cell-factor receptors. Nature $377: 242-246$

[10] Lachmann N, Ackermann M, Frenzel E et al. (2015) Large-scale hematopoietic differentiation of human induced pluripotent stem cells provides granulocytes or macrophages for cell replacement therapies. Stem Cell Reports 4:282-296

Funding: Open access funding provided by Medical University of Graz. Open Access: Dieser Artikel wird unter der Creative Commons Namensnennung 4.0 International Lizenz veröffentlicht, welche die Nutzung, Vervielfältigun erlaubt, sofern Sie den/die ursprünglichen Autor(en) und die Quelle
erenter ordnungsgemäß nennen, einen Link zur Creative Commons Lizenz beifügen und angeben, ob Änderungen vorgenommen wurden. Die in diesem Artikel enthaltenen Bilder und sonstiges Drittmaterial unterliegen ebenfalls der genannten Creative Commons Lizenz, sofern sich aus der Abbildungslegend nichts anderes ergibt. Sofern das betreffende Material nicht unter der genannten Creative Commons Lizenz steht und die betreffende Handlung nich nach gesetzlichen Vorschriften erlaubt ist, ist fur die oben aufgefuhrten Rechteinhabers einzuholen. Weitere Details zur Lizenz entnehmen Sie bitte der izenzinformation auf http://creativecommons/iz/licenses/by/4.0/deed d
Korrespondenzadresse:

Dr. med. Isabel Dorn

PD Mag. Dr. Claudia Bernecker

Universitätsklinik für Blutgruppenserologie und

Transfusionsmedizin

Auenbruggerplatz 48

A-8036 Graz

Isabel.dorn@medunigraz.at

c.bernecker@medunigraz.at

Isabel Dorn
Medizinstudium an der Medizinischen Universität zu Lübeck. 2003 Promotion am
Institut für Immunologie und Transfusionsmedizin. Facharztausbildung Transfusions-
medizin und Kinder- und Jugendmedizin an den Universitätskliniken in Lübeck,
Münster und Graz, Österreich. Forschungsaufenthalte 2010 an der Université Pierre
et Marie Curie, Paris und 2011-2015 am Max-Planck-Institut für Molekulare Biomedi-
zin, Münster. Seit 2017 an der Universitätsklinik für Blutgruppenserologie und Trans-
fusionsmedizin, Medizinischen Universität Graz.
$\begin{aligned} & \text { Claudia Bernecker } \\ & \text { Biologiestudium an der Universität Graz, Österreich. 1997-2004 Diplomarbeit am } \\ & \text { Institut für Pathophysiologie und der Abteilung für Onkologie, Medizinische Universi- } \\ & \text { tät Graz. 2006 Promotion. 2006-2012 Postdoc am Klinischen Institut für Medizini- } \\ & \text { sche und Chemische Labordiagnostik, Medizinische Universität Graz. 2014 Habilita- } \\ & \text { tion. Seit 2015 biologische Leitung der Forschungsgruppe Hematopoietic stem cell } \\ & \text { differentiation an der Universitätsklinik für Blutgruppenserologie und Transfusions- } \\ & \text { medizin, Medizinische Universität Graz. }\end{aligned}$

